\title{
The Smallest Quantum Computer Yet
}

\section{A trapped-ion-based quantum computer that fits in two boxes, each the size of a studio apartment's shower, can create a fully entangled}

\section{4-particle quantum state.}

\section{By Rachel Berkowitz}

$\square$ rototype quantum computers are beginning to surpass the computational abilities of classical computers. To achieve a true quantum advantage, these few-qubit prototypes will need to be scaled to hundreds of qubits, which will be tricky with the current laboratory-filling hardware. In a step toward accessible and scalable technology, Ivan Pogorelov at the University of Innsbruck, Austria, and colleagues have fabricated a compact quantum computing processor that fits inside two industry-standard server racks, which each have volumes of $1.7 \mathrm{~m}^{3}$ [1]. The processor performs on par with similar laboratory-scale versions, and it could eventually lead to an up-to-50-qubit device that could be operated by trained nonspecialists.

The team's design uses optical qubits that encode quantum information in two electronic states of calcium ions. Information is shared between ions, and laser pulses manipulate the ions' states and create entanglement.
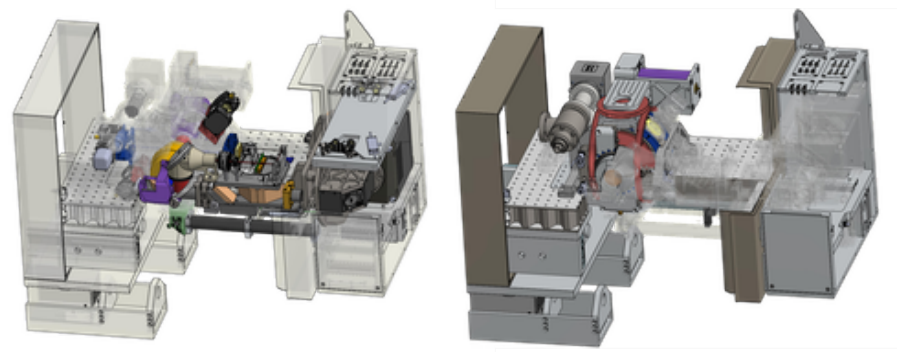

The processor relies on macroscopic electric-field traps to control strings of up to 50 ions. The system is housed in a series of sturdy aluminum boxes, called modules, that stack on top of each other inside two 19-inch-wide server racks. The "optical" rack's modules contain devices for generating and controlling laser light and for routing and switching the light. The "trap" rack's modules contain the main ion trap, electrical components for manipulating the qubits in the ions, and components for communications and remote control. The team demonstrated an ion-trap device with a fully entangled "24-qubit GHz" state, the largest fully entangled state achieved to date in any system.

The new design has easily replaceable parts and requires minimal maintenance, the team says. It can also be enabled to have cloud access for testing algorithms with a quantum computing language.

Rachel Berkowitz is a Corresponding Editor for Physics based in Vancouver, Canada.

\section{REFERENCES}

1. I. Pogorelov et al., "Compact ion-trap quantum computing demonstrator," PRX Quantum 2, 020343 (2021).

Credit: I. Pogorelov et al. [1] 